\section{SOI: 1.1/TAS DOI: $10.15863 /$ TAS International Scientific Journal Theoretical \& Applied Science}

\author{
p-ISSN: 2308-4944 (print) e-ISSN: 2409-0085 (online) \\ Year: 2015 Issue: $11 \quad$ Volume: 31 \\ Published: $30.11 .2015 \quad$ http://T-Science.org
}

SECTION 21. Pedagogy. Psychology. Innovations in the field of education.

\section{Said Abdullaevich Salekhov}

Professor, doctor of medical sciences Novgorod State University of Yaroslav Mudry,

Russia

ssalehov@mail.ru

Michail Nickolaevich Gordeev Professor, doctor of medical sciences, Institute of Psychotherapy and Clinical Psychology

Russia

mngordeev@yandex.ru

Yuliya Saidovna Salekhova

LLM

head of group of legal support WOOPPAY LLP,

Kazakhstan

salekhova.yuliya@gmail.com

Inna Alexandrovna Korabelnikova graduate student,

Novgorod State University of Yaroslav Mudry,

Russia

inna.korabelnikova@gmail.com

\title{
INFLUENCE OF EMOTIONAL AND INFORMATIONAL FACTORS IN IMPLEMENTATION OF COPING STRATEGIES IN PSYCHOLOGICAL STRESS
}

\begin{abstract}
In this article the analysis of significance of emotional pressure and informational load for implementation of coping strategy against psychological stress has been carried out. The analysis was based on fundamental provisions of psychophysiology, the theory of dominant, the theory of functional systems, regularities of development of the common adaptation syndrome, and also took into account the features of power supply for intensive nervous activity in psychological stress and A. Maslow's hierarchy of needs. When considering the psychological stress, an integrative approach to its psychological and biological interpretation was used. The accent was on the peculiarities of influence of the stress factor addressed to the central nervous system as psychological influence and power supply for response to influence of a stress factor taking into account the physiological condition of an organism. In the structure of psychological stress two factors were allocated, which are emotional pressure and cognitive loading caused by increase in the incoming information. The analysis of hierarchy of these factors based on the principle of dominant, the theory of functional systems, the biological theory of emotions and A. Maslow's hierarchy of needs allowed to prove the priority of the emotional factor, whose power requests will be satisfied first of all. It creates prerequisites for decrease in effectiveness of cognitive activity and increase of probability of making maladaptive decisions.

Key words: psychological stress, emotional pressure, informational factor, cognitive function, limbic system, principle of dominant, theory of functional systems, biological theory of emotions, A. Maslow's hierarchy of needs, coping strategies.

Language: Russian

Citation: Salekhov SA, Gordeev MN, Salekhova YS, Korabelnikova IA (2015) INFLUENCE OF EMOTIONAL AND INFORMATIONAL FACTORS IN IMPLEMENTATION OF COPING STRATEGIES IN PSYCHOLOGICAL STRESS. ISJ Theoretical \& Applied Science 11 (31): 147-154.

Soi: http://s-o-i.org/1.1/TAS-11-31-24 Doi: crossef http://dx.doi.org/10.15863/TAS.2015.11.31.24
\end{abstract}

\section{ВЛИЯНИЕ ЭМОЦИОНАЛЬНОГО И ИНФОРМАЦИОННОГО ФАКТОРОВ В РЕАЛИЗАЦИИ КОПИНГ-СТРАТЕГИЙ ПРИ ПСИХОЛОГИЧЕСКОМ СТРЕССЕ}

Аннотация: В статье, опираясь на фундаментальные положения психофизиологии, учения о доминанте, теории функциональных систем, закономерности развития общего адаптационного синдрома, а также с учетом особенностей энергетического обеспечения интенсивной нервной деятельности при 
психологической нагрузке и пирамиду потребностей А. Маслоу был проведен анализ значимости эмочионального напряжения и информационной нагрузки на реализащию копинг-стратегий на фоне психологического стресса. При рассмотрении психологического стресса был использован интегративный подход его психологической и биологической интерпретации. Акиент был на особенностях воздействия стрессора, адресованного $к$ ияентральной нервной системе как психологического воздействия и энергетического обеспечения реагирования на воздействие стрессора с учетом физиологического состояния организма. В структуре психологического стресса были выделены два фактора - это эмоциональное напряжение и интенсивная когнитивная нагрузка, обусловленная увеличением поступающей информации. Анализ иерархии этих факторов, основанный на принципе доминанты, теории функииональных систем, биологической теории эмоциии и пирамиде потребностей А. Маслоу позволил обосновать приоритетность эмочиионального фактора, энергетические запросы которого будут удовлетворяться в первую очередь. Это создает предпосылки для снижения эффективности когнитивной деятельности и возрастания вероятности принятия дезадаптивных решений.

Ключевые слова: психологический стресс, эмоциональное напряжение, информационньй фактор, когнитивная функциия, лимбическая система, принции доминанты, теория функциональных систем, биологическая теория эмоций, пирамида потребностей А. Маслоу, копинг-стратегии.

В настоящее время, отмечается неуклонный рост так называемых болезней «образа жизни», или иначе «болезней цивилизации». При этом достоверные критерии, согласно которым ту или иную патологию можно отнести к данной группе заболеваний отсутствуют.

По нашему мнению есть два показателя, позволяющие рассматривать патологию как «болезнь образа жизни», это повсеместный рост частоты выявления и тенденция к омоложению [1].

На самом деле само понятие образ жизни собирательное понятие, настолько емкое, что, по сути, и определить его достаточно сложно. С одной стороны, образ жизни рассматривается как поведенческие реакции и действия человека, зависящие от него самого: гиподинамия, пищевое поведение, употребление алкоголя и психоактивных веществ, курение, нарушение режима дня, отсутствие отдыха и нормального сна и еще многое другое. То есть, каждый сам выбирает как ему жить и что делать.

В отличие от этого, параллельно, на человека действуют факторы внешней среды, приспосабливаясь к которым, человек меняет и свой образ жизни. Так, ускоренный темп жизни, тотальная урбанизация, постоянный конфликт мотивации достижения и мотивации избегания неудач, а также целый ряд причин, вызывающих психоэмоциональное напряжение, приводят к возникновению длительных психотравмирующих ситуаций имя которым психологический стресс $[2,3,4,5]$. При этом внешние факторы оказывают значимое внимание на выбор человека как ему жить и что делать.

Если сначала стресс рассматривался с сугубо биологических позиций, то, по мере его изучения стали делать акцент на роли центральной нервной системы (ЦНС) в развитии стресса, его психологической и эмоциональной составляющей $[6,7,8,9,10]$, поскольку именно к центральной нервной системе адресованы запросы внешней среды. При этом остались без должного внимания вопросы энергетического обеспечения ответной реакции организма на предъявляемые к нему запросы стрессоров, что удовлетворяется за счет функционального состояния организма в целом.

С этих позиций психологический стресс целесообразно рассматривать, как и любую стрессовую ситуацию, без идентификации природы стрессора, то есть как общий адаптационный синдром.

В то же время, фраза Н. Selye: «Актонтребует реактона» может рассматриваться двояко. С одной стороны действительно любое воздействие (актон) вызывает ответную реакцию (реактон), а с другой, поскольку идет обсуждение стресса эту фразу рассматривают с позиции развития только общего адаптационного синдрома.

По нашему мнению это стало причиной слишком общего рассмотрения стресса как такового и желания сепарации различных специалистов различных специальностей, которые выявили отличия реакций на воздействия стрессоров в рамках своих профессиональных интересов, от феномена стресса, описанного Н. Selye [4]. Поэтому и названия стресса стали отображать либо особенности стрессора (болевой, холодовой, экзаменационный, академический, соревновательный), либо специальность, исследующую его (профессиональный, кардиологический, анестезиологический, педагогический, спортивный).

В то же время, закономерности реакции организма на воздействие стрессора стандартны, но имеет значение первичная адресация запроса к организму, к каким его органам и системам он адресован. Поэтому более правильным представляется выражение: «каждый актон требует своего реактона [1]. 
Однако, вместо интегрированного исследования феномена психологического стресса, объединив ресурсы различных дисциплин, его изучение идет параллельными курсами без сотрудничества специалистов различного профиля.

Цель: оценить значимость и влияние эмоционального напряжения и нарастающего потока информации на эффективность копинг стратегий при воздействии психологических стрессоров.

\section{Материалы и методы}

В основу данной публикации положен интегративный анализ иерархии эмоционального и информационного факторов в структуре психологического стресса опираясь на фундаментальные положения учения о доминанте А.А. Ухтомского [9] и теории функциональных систем П.К. Анохина [12, 13], его биологической теории эмоций [14], а также пирамиду потребностей А. Маслоу [15].

В основе принципа доминанты А.А. Ухтомского лежит положение о том, что при наличии приоритетного очага возбуждения в центральной нервной системе, все ресурсы направлены на удовлетворение потребностей и запросов этого очага возбуждения, а остальные отделы мозга обеспечиваются по остаточному принципу.

Согласно теории функциональных систем П.К. Анохина при воздействии, в том числе психологического фактора, формируется функциональная система, обеспечивающая адекватный ответ-реакцию на предъявляемые требования-запросы к организму, для достижения конечного положительного результата [12].

Структура и последовательность деятельности функциональной системы (рис 1.) заключается в том, что:

- при поступлении входящей афферентной информации аппарат контроля формирует модель полезного результата;

- аппарат управления, объединяющий нервные центры и эндокринные органы определяет необходимые ресурсы для достижения полезного результата;

- исполнительный аппарат, который представлен органами-аффекторами и использует выделенные ресурсы организма для реализации получения конечного полезного результата;

- аппарат контроля, по принципу обратной связи тестирует полученный результат на соответствие модели полезного результата.

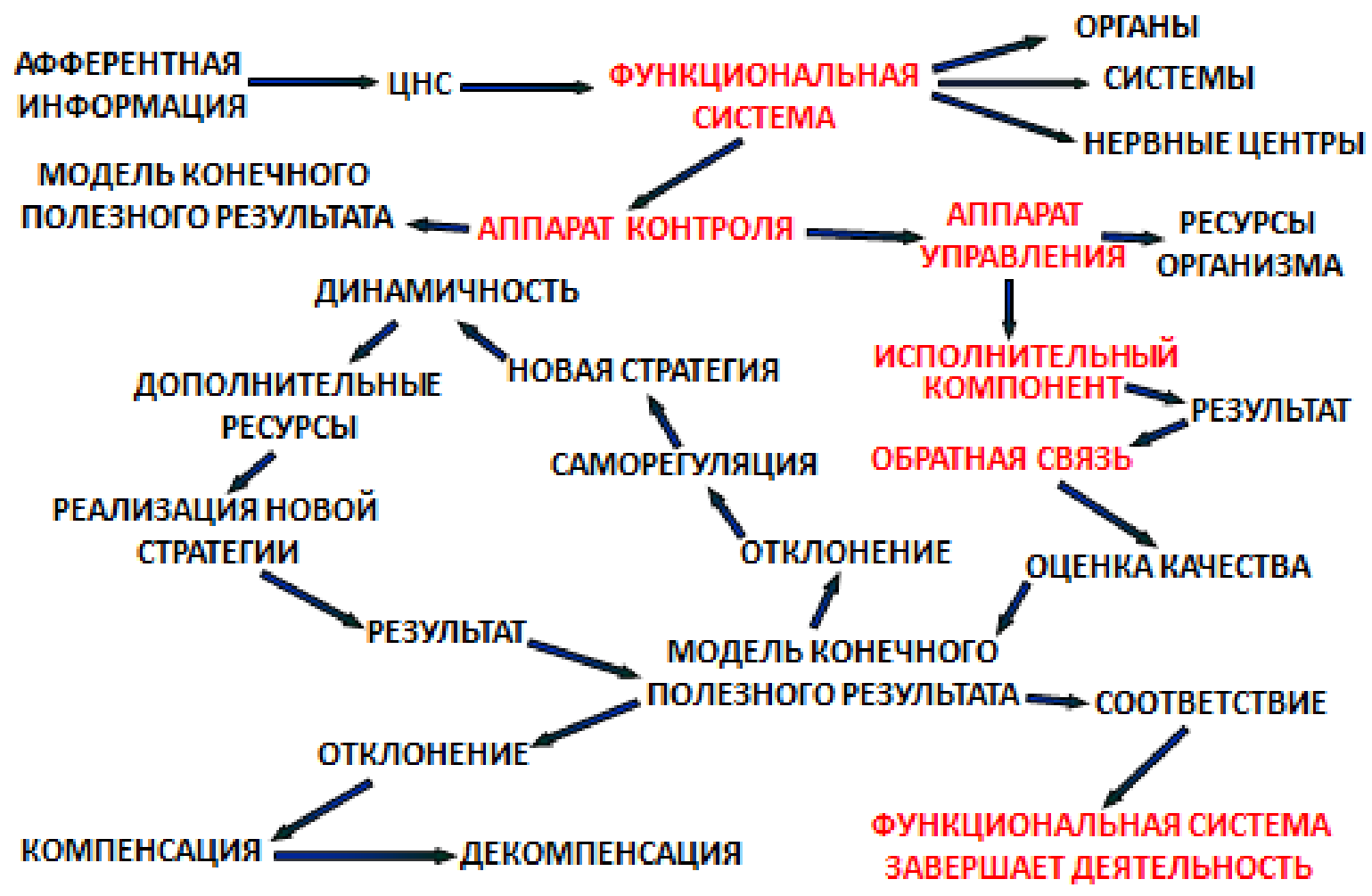

Рисунок 1 - Структура деятельности функциональной системы.

ISPC Technological advances, 
Если соответствие с моделью достигнуто функциональная система завершает свою работу, а если нет, функциональная система вырабатывает новую стратегию достижения модели полезного результата с привлечением новых ресурсов организма. Цикл достижения модели полезного результата и обратной связи повторяется снова до получения соответствия полученного результата запрограммированной модели.

При многократно повторяющихся циклах достижения полученного результата запрограммированной модели и отсутствии соответствия, включаются все новые ресурсы и компенсаторно-приспособительные механизмы, что, в конечном счете, сказывается на функциональном состоянии всего организма, может привести к истощению, декомпенсации и развитию патологических состояний. То есть, последствия деятельности функциональной системы без достижения конечного полезного результата приводят к эндогенизации патологических процессов $[1,12]$.

Согласно биологической теории, эмоции рассматриваются как биологический продукт эволюции, приспособительный фактор в жизни животных. При этом появление потребностей носит отрицательную эмоциональную окраску, играющую роль стрессора и является стимульным триггером, активизирующим организм на удовлетворение данной потребности, то есть запускает программу реагирование на воздействие стрессора [14].

При выполнения программы удовлетворения потребностей будет получен некий результат и когда обратная связь подтвердит его соответствие ожидаемому конечному полезному результаты, возникает положительная эмоция, которая при повторяющемся запросе и его удовлетворении играет не только роль положительного подкрепления, но и способствует формированию эффективной на данный момент копингстратегии, направленной на реагирование на данный запрос. При этом неэффективные стратегии будут блокироваться, а параллельно активизируется поиск эффективных стратегий удовлетворения потребностей.

В пирамиде потребностей Абрахама Маслоу выделены ступени перехода от низших биологических потребностей к высшим:

1 ступень - физиологические потребности, где стрессорами являются голод, жажда, нарушение сна, умственная и физическая усталость, чрезмерно быстрый темп жизни;

2 ступень - потребности безопасности и стабильности, где к стрессорам относятся страх и тревога потерять работу, провала на экзамене, страх за жизнь близких, страх смерти и т.д.;
3 ступень - потребность в принадлежности и любви, где стрессорами являются моральное и физическое одиночество, потеря близких людей или болезнь, неразделенная любовь;

4 ступень - потребность в уважении, где стрессорами будут крушение карьеры, провал на экзамене, невозможность реализовать свои амбиции, потеря уважения в обществе;

5 ступень - потребность в самореализации, где причинами стресса будут невозможность реализовать свои способности, вмешательство со стороны родителей или факторов внешней среды, которые препятствуют занятиям любимым делом, следовать своему признанию.

При этом психологический стресс относится ко второй (безопасность и стабильность) и четвертой (уважение, признание, самореализация, страх быть отвергнутым обществом и потерять уважение) ступени [15].

Определение места эмоционального и информационного фактора в пирамиде потребностей А. Маслоу позволяет определить их значимость для организма и определить иерархию в структуре психологического стресса.

Кроме того, был использован интегративный подход, объединяющий преимущества психологической и биологической интерпретации стресса, с учетом точки воздействия стрессора и энергетическое обеспечение ответной реакции на это воздействие.

\section{Результаты исследования}

В соответствии с принципом доминанты А.А. Ухтомского в первую очередь будут удовлетворяться запросы либо эмоционального, либо информационного фактора, в зависимости от приоритетности для организма. При этом эмоциональный и информационный фактор конкурируют за обладание ресурсами организма, в первую очередь за обладание энергетическим субстратом, позволяющим эффективно удовлетворить запрос стрессора.

Обработка входящей информации и формирование на нее адекватного ответа происходит под контролем ЦНС, обеспечивающей регуляцию взаимодействия функциональных систем, определении приоритетности и последовательности достижения конечных положительных результатов, в соответствии с принципами конвергенции (концентрация поступающей информации из разных зон в одном центре), иррадиации (распространении информации из одного центра на другие зоны участвующие в получении конечного полезного результата), реципрокности (синхронизации возбужденияторможения в нервных центрах-антогонистах), доминанты (приоритетности решения конкретной задачи), обратной связи (тестировании полученного результата с конечным полезным 
результатом) и субординации (подчиненность низших отделов нервной системы высшим, которым является кора больших полушарий).

При этом большую роль в обеспечении регуляторной функции ЦНС играет гипоталамогипофизарная система, которая считается преобразователем входящей нервной информации, которая приходит от различных участков мозга в гормоны и биологически активные вещества, которые могут регулировать деятельность внутренних органов человека. При этом ретикулярная формация играет очень важную роль в передаче психофизиологической информации, поскольку она получает всю информацию, которая поступает от рецепторов и направляет еe в соответствующий участок головного мозга [8].

Следует отметить, что при активности какой-либо функциональной системы, которая становится доминантной в данный момент времени, остальные функциональные системы становятся второстепенными и находятся в рецессивном положении. Более того, функциональная система, даже при ее приоритетности, если она включена в процесс реагирования, которую обеспечивает, в том числе, и уже активная функциональная система становится на время рецессивной, пока не будет завершена деятельность доминантной, активной на данный момент времени, функциональной системы.

Согласно модели стресса приведенной Дж. С. Гринбергом сначала появляется жизненная ситуация, которая воспринимается нами как стрессовая. Затем в ответ на эту стрессовую ситуацию сначала развивается эмоциональное возбуждение, которое затем приводит к физиологическому возбуждению. Только после реализации этой цепочки появляются последствия стресса как такового [9].

Это совпадает и с особенностями транспорта информации по проводящим путям. Так, как входящая афферентная информация, так и исходящая эфферентная проходят через лимбическую систему, которая отвечает за формирование эмоций. При этом пока информация поступит в лобные доли, а затем сформулированный ответ начнет передаваться к периферическим рецепторам, в лимбической системе уже появится доминантный очаг возбуждения, поскольку будет происходить эмоциональное реагирование на входящую информацию.

Психологический стресс занимает вторую и четвертую ступень в пирамиде потребностей А. Маслоу [15]. Поэтому для определения иерархии эмоционального и информационного фактора в структуре психологического стресса необходимо определить место каждого из них в пирамиде потребностей А. Маслоу [16]. При этом следует учитывать, что наиболее приоритетными, обеспечивающими выживание организма являются первая и вторая ступени, которые удовлетворяют физиологические потребности и обеспечивают безопасность и стабильность.

По сути своей воздействие этих факторов кардинально различается между собой, хотя точка приложения едина, это центральная нервная система (ЦНС). При этом воздействие адресовано к различным отделам ЦНС: психоэмоциональный фактор (неспецифическая информация о приближающейся стрессовой ситуации) адресован и воздействует на ответственную за формирование эмоций лимбическую систему, а информационный фактор (большой объем информации, который необходимо осмыслить, определить и выбрать оптимальную стратегию реагирования) действующий на ответственную за когнитивную функцию кору больших полушарий.

Учитывая, что во время психологического стресса имеет место психоэмоциональная напряженность, которая замыкается на лимбической системе головного мозга, проявлениями которой являются тревожность и страх, нарушение сна, психоэмоциональная лабильность, раздражительность, а порой и агрессия, эмоциональная составляющая определяется реализацией рефлекса «борьбабегство», занимающего вторую ступень в пирамиде потребностей А. Маслоу.

По мнению Lazarus R.S. (1977), эмоции, в сочетании $\mathrm{c}$ когнитивной функцией, имеют большое значение в формировании копингстратегий при воздействии стрессора [17].

В отличие от этого, способность выбрать оптимальное решение в конкретной ситуации, ее анализ и прогнозирование сценариев развития, варианты своих действий и предположительная оценка их эффективности, оценочное восприятие окружающими этой ситуации и правильности твоих решений относится к информационной составляющей психологического стресса и во многом зависит от состояния когнитивной функции,

Нарушения когнитивной функции во время стрессовой ситуации определяют как самооценку, так и оценку со стороны окружающих, что соответствует четвертой ступени в пирамиде потребностей А. Маслоу. Более того, нарушения когнитивной функции имеет еще и эмоциональную окраску, что может еще больше усугубить нарушение когнитивной функции.

То есть эмоциональный фактор в пирамиде потребностей А. Маслоу занимает предпочтительную позицию, по сравнению с информационным. 
Если оценивать временную активацию психоэмоционального и информационного факторов в структуре психологического стресса с позиции формирования приоритетности в соответствии с принципом доминанты А.А. Ухтомского видно, что задолго до развития стрессовой ситуации, когда только поступает минимальная информация о ее вероятности, без конкретной информации на которую надо сразу реагировать появляется безадресная тревожность, постепенно нарастающая по мере приближения воздействия стрессора.

На этом фоне создаются предпосылки для формирования застойного очага возбуждения в лимбической системе, который становится доминантным и приоритетным по удовлетворению своих запросов в отношении энергетического и пластического обеспечения.

Соответственно, когда возникает стрессовая ситуация с предъявлением конкретного информационного запроса к организму, требующего когнитивного анализа ситуации, он удовлетворяется по остаточному принципу.

Если оценивать психоэмоциональный фактор, а именно, тревожность, появляющуюся задолго до реальной стрессовой ситуации, в виде безадресного неконкретного запроса к организму в поддержании его в состоянии готовности для реакции в любой текущий момент времени, то с позиции теории функциональных систем П.К. Анохина, становится понятным появление такого проявления эмоциональной напряженности как нарушение сна. При этом нарушение сна в пирамиде А. Маслоу занимает первую ступень и относится к физиологическим потребностям.

То есть, в соответствии с принципом доминанты А.А. Ухтомского и основными положениями теории функциональных систем П.К. Анохина эмоциональный фактор, на фоне последствий реакции на него организма в пирамиде потребностей А. Маслоу определяет не только безопасность и стабильность (вторая ступень), но и физиологические потребности организма (первая ступень), что делает его безусловно приоритетным по сравнению с информационным фактором.

В то же время, отсутствие конкретного запроса на удовлетворение актуальной потребности, а подмена его на ожидаемое воздействие в будущем, в соответствии с биологической теорией эмоций П.К. Анохина [14] будет способствовать не только появлению отрицательных эмоций, но и, вследствие отсутствия удовлетворения в течение длительного времени этой безадресной потребности, приводить к истощению адаптивных ресурсов и ЦНС, и всего организма в целом.

Соответственно, особенностью реакции на психологический стрессор будет то, что афферентная информация к высшим отделам ЦНС, а затем эфферентная от центра к периферии проходит через лимбическую систему (рис 2).

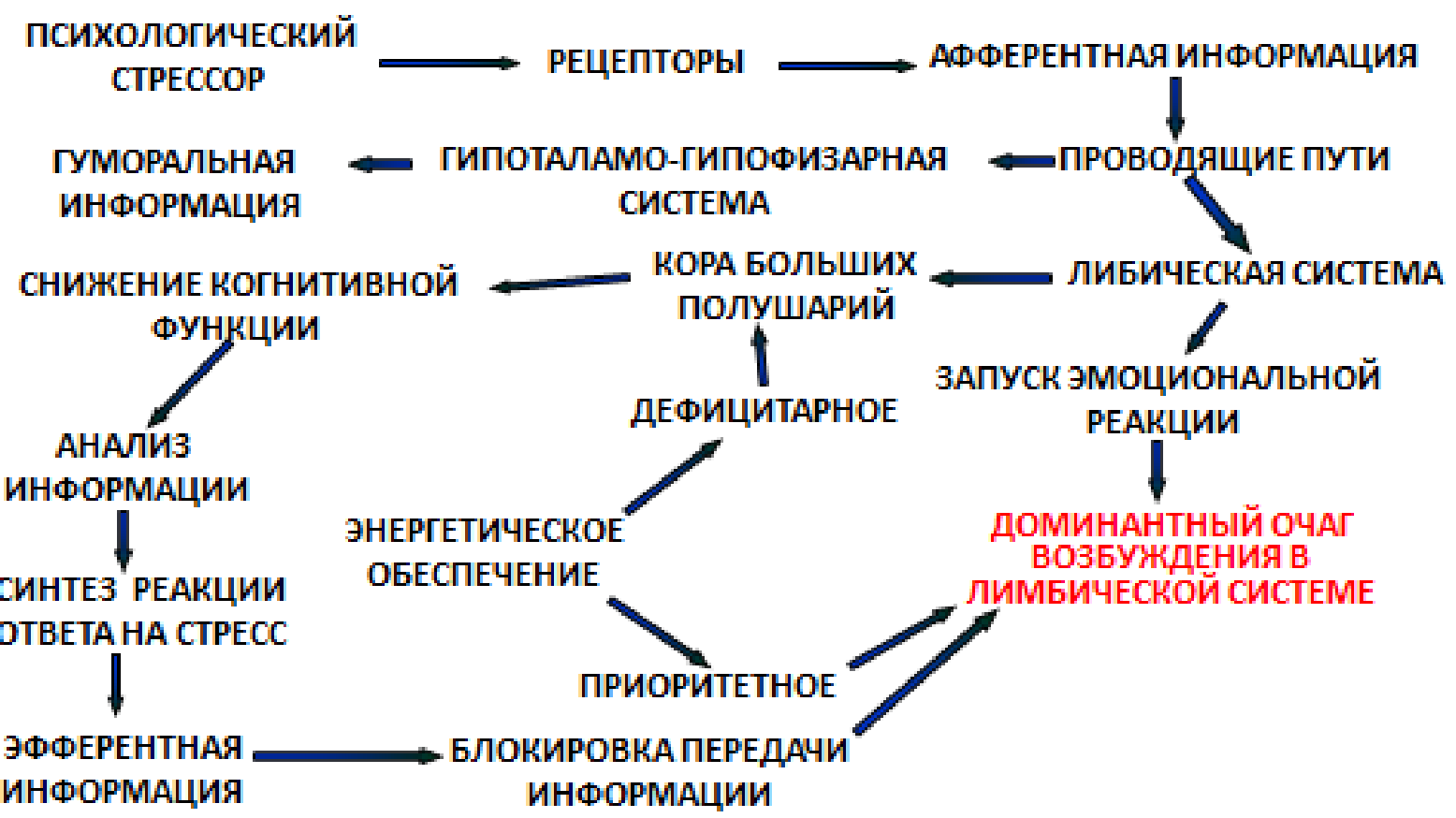

Рисунок 2 - Схема реакции на воздействие психологического стрессора.

ISPC Technological advances, 
Появление очага возбуждения в виде эмоций, генерируемых лимбической системой в ответ на первичное воздействие может заблокировать транспорт информации как к ЦНС, так и от нее к периферии, поскольку в соответствии с доминантой А.А. Ухтомского удовлетворяться будет энергетическое обеспечение поддержания эмоций, поскольку функциональная система, обеспечивающая их стала приоритетной как за счет более раннего старта, так и того, что приспособительная функция эмоций относится к обеспечению безопасности и стабильности в режиме здесь и сейчас.

В отличие от этого, за счет более раннего начала деятельности функциональной системы, обеспечивающей эмоции, когнитивная функция будет обеспечиваться в ущербном варианте по остаточному принципу. При этом очаг возбуждения в лимбической системе, обеспечивающей проявление эмоций, все свои ресурсы будет расходовать на это, а проведение как афферентной, так и эфферентной информации будет снижено, либо нарушено в полной мере. Соответственно, транспорт информации от ЦНС к периферии через лимбическую систему будет заблокирован.

Использование когнитивной функции позволяет выбрать более эффективную реакцию на воздействие психологического стрессора, но эмоциональная реакция на него может привести к блоку когнитивного осмысления стрессовой ситуации и реагирования на нее по типу «борьбабегство» [18].

Таким образом, эмоциональный фактор является приоритетным в структуре психологического стресса, а его запросы будут удовлетворяться в режиме здесь и сейчас, по сравнению с ущербным отсроченным удовлетворением запросов информационного фактора и когнитивной функции. На этом фоне создаются предпосылки для снижения эффективности и нарушения когнитивной функции, следствием чего может быть принятие дезаптивных решений

Соответственно, функциональная система, обеспечивающая удовлетворение запроса доминантного очага возбуждения психоэмоционального стресса, согласно принципу доминанты [11] и теории функциональных систем [12] будут блокировать функциональную активность функциональной системы, направленной на удовлетворение информационного компонента в структуре психологического стресса.

Дефицит энергетического обеспечения обработки информации при воздействии психологического стрессора будет приводить к принятию дезадаптивных решений. То есть, недостаток энергетического обеспечения будет снижать эффективность не только обработки входящей информации и ее перевода из короткой в долговременную память, но и к снижению эффективности, либо нарушению извлечения необходимых данных из долговременной памяти. В совокупности это создает предпосылки для снижения эффективности, а иногда и нарушению когнитивной функции, следствием чего может быть принятие не эффективных решений.

\section{References:}

1. Salehov SA (2014) Psihoemotsionalnaya informatsionno-energeticheskaya teoriya ozhireniya /S. A. Salehov; Velikiy NovgorodAlmatyi, 2014. - 180 p.

2. Shear MK (1986) Pathophysiology of panic: a review of pharmacologic provocative test and naturalistic monitoring data. / M.K Shear // J. Clin-Psych. 1986. Vol.47. pp. 18 - 26.

3. Selye H (1938) Adaptation Energy // Nature 141, $926 \quad$ (21 May 1938) doi:10.1038/141926a0

4. Selye H (1936) A Syndrome produced by Diverse Nocuous Agents. // Nature 138, 32 (4 July 1936) | doi:10.1038/138032a0.
5. McEwen BS (2013) The Brain on Stress: Toward an Integrative Approach to Brain, Body and Behavior. // Perspect Psychol Sci. 2013 Nov 1;8(6):673-675.

6. (2006) Psihofiziologiya: Uchebnik dlya vuzov pod red. YuI Aleksandrova. SPb., 2006. - 331 p.

7. Vilyunas VK (1984) Osnovnyie problemyi psihologicheskoy teorii emotsiy // Psihologiya emotsiy: Tekstyi. - M., 1984. - pp. 3-28.

8. Gordeev MN (2008) NLP v psihoterapii: M.: «Psihoterapiya», 2008. - $272 \mathrm{p}$.

9. Grinberg DzhS (2002) Upravlenie stressom. / 7e izd. - SPb.: Piter, 2002. - 496 p. 


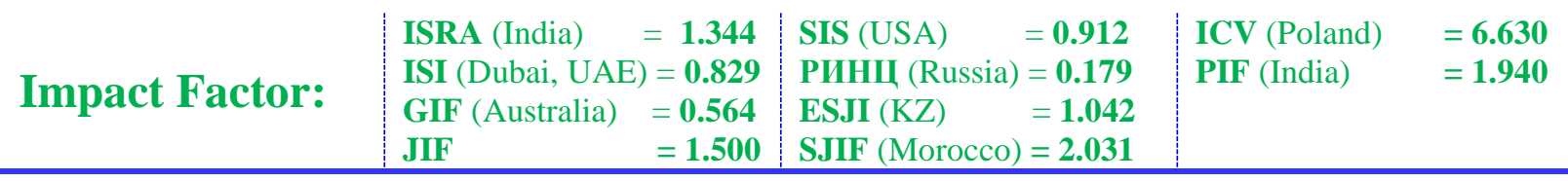

10. Richardson M, Abraham C, Bond R (2012) Psychological correlates of university students' academic performance: A systematic review and meta-analysis // Psych Bul. - 2012, Vol 138(2), pp. 353-387.

11. Uhtomskiy AA (1966) Dominanta / AA Uhtomskiy / M. - L.: Nauka, 1966. 273 p.

12. Anohin PK (1970) Teoriya funktsionalnoy sistemyi / PK Anohin / Uspehi fiziol. nauk. 1970. T. 1, \# 1. pp. 19 - 54.

13. Salehov SA (2013) Psihofiziologicheskie osobennosti podstroyki dyihaniya pri ustanovlenii rapporta. Monografiya /SA Salehov; Novgorodskiy gosudarstvennyiy universitet imeni Yaroslava Mudrogo. - Velikiy Novgorod, 2013. - 144 p.

14. Anohin PK (1964) Emotsii // Bolshaya meditsinskaya entsiklopediya. T. 35. - M., 1964. - pp. 339.

15. Scherbatyih YV (2012) Psihologiya stressa i metodyi korrektsii. 2-e izd. - SPb.: Piter, 2012. $-256 \mathrm{p}$.
16. Salehov SA, Gordeev MN, Bizina TV, Maksimyuk NN (2015) Ierarhiya psihoemotsionalnogo i informatsionnogo faktorov v strukture ekzamenatsionnogo stressa // Sovremennyie problemyi nauki i obrazovaniya. - 2015. - \# 5; Available: http://www.science-education.ru/128-21959 (Accessed: 10.11.2015).

17. Lazarus RS (1977) Cognitive and coping processes in emotion. / / Stress and coping. N.Y., Columbia Univ. Press, 1977, pp. 144-157.

18. Salekhov SA, Esaulov VI, Yablochkina SO (2015) INFLUENCE OF EXPECTANT MOTHERS NEGATIVE STRESS ON COPING STRATEGY AND THEIR ENERGY SUPPLY FORMING. ISJ Theoretical \& Applied Science 10 (30): 111-116. Soi: http://so-i.org/1.1/TAS-10-30-24 Doi: http://dx.doi.org/10.15863/TAS.2015.10.30.24 